\title{
Strong spatial dispersion in wire media in the very large wavelength limit
}

\author{
P. A. Belov,${ }^{1,2}$ R. Marqués, ${ }^{3}$ S. I. Maslovski, ${ }^{1}$ I. S. Nefedov ${ }^{1,4}$ M. Silveirinha, ${ }^{5}$ C. R. Simovski, ${ }^{1,2}$ and S. A. Tretyakov ${ }^{1}$ \\ ${ }^{1}$ Radio Laboratory, Helsinki University of Technology, P.O. Box 3000, FIN-02015 HUT, Finland \\ ${ }^{2}$ Physics Department, St. Petersburg Institute of Fine Mechanics and Optics, Sablinskaya 14, 197101 St. Petersburg, Russia \\ ${ }^{3}$ Department of Electronics and Electromagnetism, Facultad de Física, University of Sevilla, \\ Avenida Reina Mercedes s/n, 41012 Sevilla, Spain \\ ${ }^{4}$ Institute of Radio Engineering and Electronics, Russian Academy of Science, Zelyonaya 38, 410019 Saratov, Russia \\ ${ }^{5}$ Instituto Superior Técnico, Instituto de Telecomunicações, Avenida Rovisco Pais, 1049-001 Lisboa, Portugal
}

(Received 27 November 2002; published 25 March 2003)

\begin{abstract}
It is found that there exist composite media that exhibit strong spatial dispersion even in the very large wavelength limit. This follows from the study of lattices of ideally conducting parallel thin wires (wire media). In fact, our analysis reveals that the description of this medium by means of a local dispersive uniaxial dielectric tensor is not complete, leading to unphysical results for the propagation of electromagnetic waves at any frequencies. Since nonlocal constitutive relations have been usually considered in the past as a secondorder approximation, meaningful in the short-wavelength limit, the aforementioned result presents a relevant theoretical interest. In addition, since such wire media have been recently used as a constituent of some discrete artificial media (or metamaterials), the reported results open the question of the relevance of the spatial dispersion in the characterization of these artificial media.
\end{abstract}

DOI: 10.1103/PhysRevB.67.113103

PACS number(s): 78.20.Ci, 41.20.Jb, 42.70.Qs, 78.70.Gq

Causality imposes that all material media must be dispersive. In most cases this behavior results in local dispersive constitutive relations, i.e., in frequency-dependent constitutive permittivity and permeability tensors. Nonlocal dispersive behavior (i.e., spatial dispersion), which results in constitutive operators depending also on the spatial derivatives of the mean fields (or, for plane electromagnetic waves, on the wave-vector components), is usually considered as a small effect, meaningful in the short-wavelength limit. Specifically, spatial dispersion will always appear when the higher-order terms in the series expansion of the constitutive parameters in power series of the dimensionless parameter $a / \lambda$ ( $a$ is the lattice constant of the crystal and $\lambda$ the wavelength inside the medium) are not neglected. ${ }^{1}$ Thus, it is usually assumed that nonlocal dispersive reations are only meaningful when $\lambda$ approaches $a$. The usually weak natural optical activity of some materials is a well-known example of the application of this principle. ${ }^{1}$ When such principle is translated to the analysis of discrete artificial media, also called metamaterials, it would imply that nonlocal dispersive constitutive relations are only expected to be a small refinement of the local constitutive relations usually considered. However, there is at least a counterexample for this assumption.

The parallel wire medium is a medium formed by a regular lattice of ideally conducting wires with small radii compared to the lattice periods and the wavelength, see Fig. 1. It has been known in microwave applications for a long time ${ }^{2-4}$ as an artificial dielectric, also called rodded medium and quasistatic models of the effective permittivity are available. ${ }^{5}$ Recently, some attention to wire media has been paid also in optics (e.g., Refs. 6,7) and in the realization of left-handed media ${ }^{8,9}$ as composite media made from lattices of long conducting wires and split ring resonators ${ }^{10-12}$ (see discussions corresponding to that $\mathrm{in}^{13}$ ). The electromagnetic reponse of the specific wire medium shown in Fig. 1 is analyzed in
Refs. 3,7 following different approaches. Both analysis are carried out for wave propagation perpendicular to the wires and show that, for electric-field polarization along the wires, the medium is characterized (if $a / \lambda, b / \lambda \ll 1$ ) by a frequencydependent effective dielectric constant given by

$$
\varepsilon=\varepsilon_{0}\left(1-\frac{k_{0}^{2}}{k^{2}}\right)=\varepsilon_{0}\left(1-\frac{\omega_{0}^{2}}{\omega^{2}}\right)
$$

The constant $\omega_{0}$ (the corresponding wave number $k_{0}$ $=\omega_{0} \sqrt{\varepsilon_{0} \mu_{0}}$ ) in Eq. (1) plays the role of an equivalent "plasma frequency." Thus, this medium is often called "artificial plasma" since the ideal (collisionless) electron plasma is described by the same equivalent parameter. If the wires

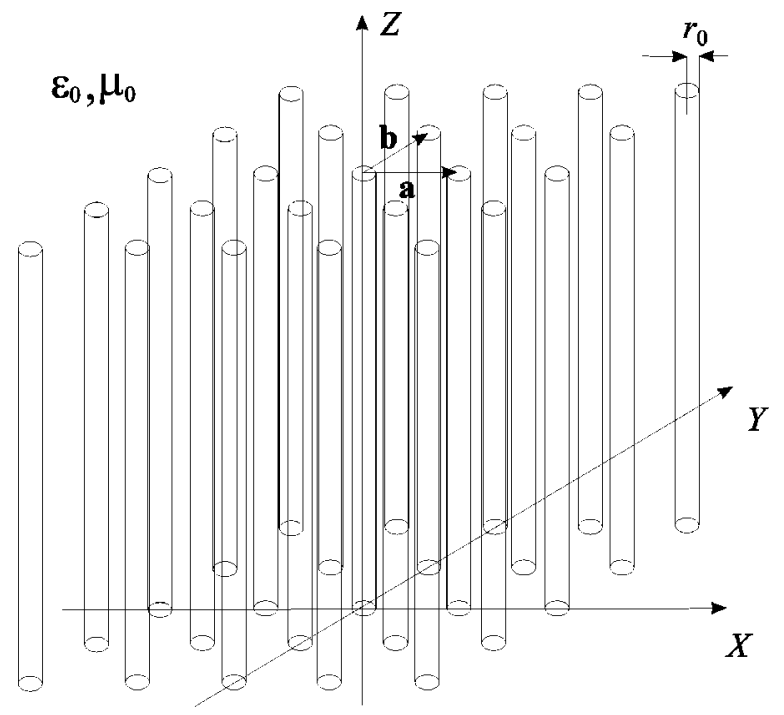

FIG. 1. The geometry of wire media: A lattice of parallel ideally conducting thin wires. 
are assumed to be very thin, so that their polarization in the direction orthogonal to the wires can be neglected, the effective permittivity for electric-field polarization orthogonal to the wires is $\varepsilon_{0}$.

The aforementioned analysis suggests that this wire medium could be modeled as a uniaxial dielectric with the following local permittivity dyadic:

$$
\overline{\bar{\varepsilon}}=\varepsilon \mathbf{z}_{0} \mathbf{z}_{0}+\varepsilon_{0}\left(\mathbf{x}_{0} \mathbf{x}_{0}+\mathbf{y}_{0} \mathbf{y}_{0}\right),
$$

whose permittivity in the axial direction, $\varepsilon$, would be given by Eq. (1). However, it will be shown in the following that this naive hypothesis leads to unphysical results and must be substituted by a nonlocal dispersive relation. In fact, assuming that the medium can be described by the uniaxial dyadic (2), the dispersion equation for extraordinary plane waves $\left(E_{z} \neq 0\right)$ with the wave vector $\left(q_{x}, q_{y}, q_{z}\right)^{\mathrm{T}}$ in this uniaxial dielectric reads ${ }^{14,15}$

$$
\varepsilon_{0}\left(q_{x}^{2}+q_{y}^{2}\right)=\varepsilon\left(k^{2}-q_{z}^{2}\right),
$$

where $k=\omega \sqrt{\varepsilon_{0} \mu_{0}}$ is the phase constant of the host matrix. On the other hand, these extraordinary waves correspond to the well-known TM (to $z$ ) set of modes, allowed by the invariance of the boundary conditions along $z$. Thus, for any extraordinary wave traveling with a phase constant $q_{z}$ along the $z$ axis, the $E_{z}$ field must satisfy the Helmholtz equation

$$
\left\{\frac{\partial}{\partial x^{2}}+\frac{\partial}{\partial y^{2}}+\left(k^{2}-q_{z}^{2}\right)\right\} E_{z}=0,
$$

with the boundary condition $E_{z}=0$ on the wires. It is clear from this equation that any "plane" extraordinary wave must satisfy

$$
k\left(q_{x}, q_{y}, q_{z}\right)=\sqrt{k^{2}\left(q_{x}, q_{y}, 0\right)+q_{z}^{2}} .
$$

This last result is incompatible with Eq. (1)-(3), as can be easily seen by substitution of Eq. (1) into Eq. (3). However, if we choose

$$
\varepsilon\left(k, q_{z}\right)=\varepsilon_{0}\left(1-\frac{k_{0}^{2}}{k^{2}-q_{z}^{2}}\right)
$$

instead of Eq. (1), then Eqs. (2) and (3) become compatible with Eq. (5), giving the following dispersion equation for the plane wave:

$$
q^{2} \equiv q_{x}^{2}+q_{y}^{2}+q_{z}^{2}=k^{2}-k_{0}^{2},
$$

where we have assumed that $q_{z} \neq k$ (the case with $q_{z}=k$ will be analyzed at the end of this paper). The above rationale suggests that the considered wire media still can be described by the permittivity dyadic (2), but the axial permittivity $\varepsilon$ must be a nonlocal parameter of the form (6). The conventional expression (1) would be only a particular case of Eq. (6), valid for wave propagation in the $x-y$ plane.

The main difference between the local uniaxial model, Eq. (1), and the proposed nonlocal model, Eq. (6), for the parallel wire medium is that the nonlocal model predicts a stop band (at frequencies below $\omega_{0}=k_{0} / \sqrt{\varepsilon_{0} \mu_{0}}$ ) for extraor- dinary waves propagating along any direction in the media. On the contrary, Eqs. (1) - (3) predict propagation of extraordinary waves at any frequency provided $q_{z}>k=\omega \sqrt{\mu_{0} \varepsilon_{0}}$. Thus, both models predict qualitatively very different behaviors, even near the cutoff plasma frequency $\omega_{0}$ where $q^{2}$ $\rightarrow 0$ (i.e., $a / \lambda \rightarrow 0$ ). That is, the nonlocality of the proposed constitutive relations affects the electromagnetic response of the medium even in the very large wavelength limit, thus being important for any values of the $a / \lambda$ ratio inside the medium. Other relevant differences between the predictions of both models will be developed along this paper.

The rigorous proof of Eq. (6) is based on the local-field approach which is described in detail in Ref. 16. In Ref. 16 the low-frequency stop band of the wire medium has been analyzed, as well as its high-frequency band-gap structure. This analysis reveals that, in the thin wire medium (Fig. 1) and for $q_{z} \neq k$, two sets of modes can propagate: ordinary (with $E_{z}=0$ ) and extraordinary (with $E_{z} \neq 0$ ) waves. The ordinary waves do not interact with the wires and propagate in the host media. For extraordinary waves, an explicit dispersion equation connecting the wave vector $\mathbf{q}$ $=\left(q_{x}, q_{y}, q_{z}\right)^{\mathrm{T}}$ with the wave number of the host isotropic matrix $k=\omega \sqrt{\varepsilon_{0} \mu_{0}}$ has been derived in Ref. 16. It can be written as

$$
\begin{aligned}
\frac{1}{\pi} \ln \frac{b}{2 \pi r_{0}} & +\frac{1}{b k_{x}^{(0)}} \frac{\sin k_{x}^{(0)} a}{\cos k_{x}^{(0)} a-\cos q_{x} a} \\
& +\sum_{n \neq 0}\left(\frac{1}{b k_{x}^{(n)}} \frac{\sin k_{x}^{(n)} a}{\cos k_{x}^{(n)} a-\cos q_{x} a}-\frac{1}{2 \pi|n|}\right)=0 .
\end{aligned}
$$

Here $k_{x}^{(n)}$ denotes the $x$ component of $n$th Floquet mode wave vector:

$$
k_{x}^{(n)}=-j \sqrt{\left(q_{y}+\frac{2 \pi n}{b}\right)^{2}+q_{z}^{2}-k^{2}}, \quad \operatorname{Re}\{\sqrt{()}\}>0 .
$$

The other notations are clear from Fig. 1. Numerical solution of this dispersion equation shows that there exists a lowfrequency stop band for all propagation directions (except for the particular case of $q_{z}=k$ that will be analyzed later). Let us simplify the dispersion equation (8) for the quasistatic case $a, b \ll \pi / k$. Using the Taylor expansion of $\sin (x)$ and $\cos (x)$ functions for small arguments we obtain Eq. (7) with

$$
k_{0}^{2}=\frac{2 \pi / s^{2}}{\ln \frac{s}{2 \pi r_{0}}+F(r)},
$$

where $s=\sqrt{a b}, r=a / b$, and

$$
F(r)=-\frac{1}{2} \ln r+\sum_{n=1}^{+\infty}\left(\frac{\operatorname{coth}(\pi n r)-1}{n}\right)+\frac{\pi r}{6} .
$$

Therefore, we have shown the suitability of the suggested approach for the description of the wire medium, with $k_{0}$ given by Eq. (10). Parameter $k_{0}$ here plays the role of the 
wave number corresponding to the plasma frequency. More exactly, it indicates the upper edge of the low-frequency stop band. Naturally, $k_{0}$ as a function of two lattice periods $a$ and $b$ is a symmetric function: $k_{0}(a, b)=k_{0}(b, a)$. It means that function $F(r)$ has the following property: $F(1 / r)=F(r)$. For the commonly used case of the square grid $(a=b), F(1)$ $=0.5275$. Expression (10) looks similar to the approximate expressions for the plasma frequency developed earlier in Refs. 2-4,6, but for thin wires it is more accurate and takes into account the geometry of the lattice. Notice that the dispersion equation (7) for extraordinary waves is indifferent to the direction of the wires' axis: the wave-vector components $q_{x}, q_{y}, q_{z}$ enter into this equation completely symmetrically. It means that, within the low-frequency stop band, the extraordinary wave decays with the same decay factor along all directions in space. The same can be said for propagation in the first frequency passband. This isotropy of the dispersion equation is rather surprising since the medium is strongly anisotropic. However, it can be shown from the very fundamental facts summarized in Eqs. (5) and (3) by assuming, as usual, that Eq. (1) is valid for extraordinary waves propagating in the $x-y$ plane.

It is possible to transit Eq. (6) from the spectral domain $(\mathbf{q}, \omega)$ to the physical domain $(\mathbf{r}, t)$. The following nonlocal material equation can be derived from Eq. (6) using the double Fourier transform:

$$
\begin{aligned}
\mathbf{D}(x, y, z)= & \varepsilon_{0} \mathbf{E}(x, y, z)+\frac{\varepsilon_{0} k_{0}^{2} c}{2} \mathbf{z}_{0} \int_{-\infty}^{t} \int_{z-c\left(t-t^{\prime}\right)}^{z+c\left(t-t^{\prime}\right)} \\
& \times E_{z}\left(x, y, z^{\prime}, t^{\prime}\right) d z^{\prime} d t^{\prime},
\end{aligned}
$$

where $c=1 / \sqrt{\varepsilon_{0} \mu_{0}}$ is the speed of light in the host matrix. Here, the area of integration in the $z-t$ plane is the light cone $\left|z-z^{\prime}\right|<c\left(t-t^{\prime}\right)$. In other words, the kernel in the Fourier convolution is $u\left[c\left(t-t^{\prime}\right)-\left|z-z^{\prime}\right|\right]$, where $u(x)$ is the Heaviside step function. It means that the point $(x, y, z)$ inside the wire medium (described as a dispersive continuum) at moment $t$ is affected by the $z$ components of electric fields coming from the domain $\left(x, y, z \pm c\left(t-t^{\prime}\right)\right)$ surrounding (along the wire axis) this point during all the past time $\left(t^{\prime}\right.$ $<t$ ). This result illustrates the consistency of the reported model from the relativistic standpoint.

In the following we will describe some relevant effects in the analyzed parallel wire medium, associated with the spatial dispersion. Refraction and reflection of plane waves at a plane interface show strong differences between the local and nonlocal models. Let us consider an interface between an isotropic dielectric with the permittivity $\varepsilon_{1}$ and a uniaxial dielectric with $\varepsilon$ described by Eq. (1). The interface is in the $y-z$ plane and it is illuminated by a plane wave coming from the isotropic dielectric. The wave vector and electric-field vector lie in $x-z$ plane $\left(q_{y}=0, E_{y}=0\right)$. The incidence angle of the plane wave is $\theta$. If $\varepsilon_{1}>\varepsilon_{0}, \varepsilon<0$, and $\sin ^{2}(\theta)$ $<\varepsilon_{0} / \varepsilon_{1}$ the wave will be completely reflected, but for $\sin ^{2}(\theta)>\varepsilon_{0} / \varepsilon_{1}$ some part of the wave will be transmitted through the interface. This transmitted wave will be an extraordinary wave, as it follows from its polarization state, and can be excited at any frequency. This amazing behavior

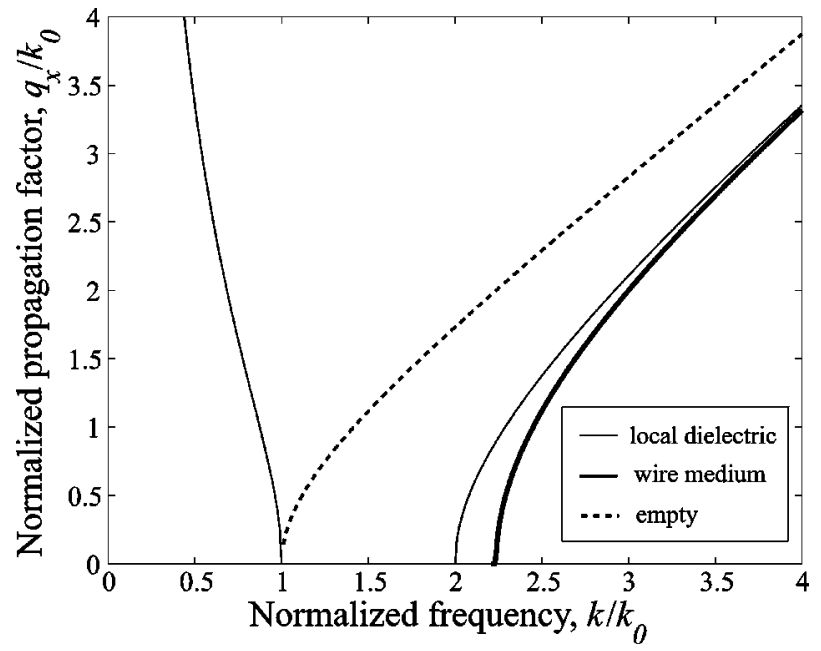

FIG. 2. Normalized propagation factors in a parallel-plate waveguide vs normalized frequency for different types of waveguide filling: Thin solid lines, uniaxial dielectric with a negative permittivity; thick solid line, wire medium; dashed line, empty waveguide. The wire medium and the uniaxial dielectric have the same $k_{0}$ $=\pi /(2 d)$.

disappears when the nonlocal model summarized in Eq. (6) is used. Indeed, if the nonlocal axial permittivity (6) is used for the wire medium, we observe that no transmission inside the wire medium is possible for $k<k_{0}$. At $k=k_{0}$ transmission is possible only in the case of the normal incidence. Only if $k>k_{0}$, a refracted wave appears.

Let us next consider the guidance of electromagnetic waves in a parallel-plate waveguide infinite in the $x$ and $y$ directions and bounded by parallel perfectly conducting planes orthogonal to the $z$ axis. Separation between the conducting walls is $d$. We assume that this waveguide is filled with a wire medium with the wires along the $z$ direction. We will consider eigenwave propagation along the $x$ axis of the $\mathrm{TM}_{01}$ mode $\left(H_{y}, E_{x}, E_{z} \neq 0\right)$. For waveguides filled by a local uniaxial dielectric with anisotropy axis along the $z$ direction we have from Eq. (3)

$$
\varepsilon_{0} q_{x}^{2}=\varepsilon\left(k^{2}-q_{z}^{2}\right), \quad q_{x}=\sqrt{\frac{\varepsilon}{\varepsilon_{0}}\left[k^{2}-\left(\frac{\pi}{d}\right)^{2}\right]} .
$$

If $\varepsilon>0$, Eq. (13) gives a cutoff for $k<\pi / d$ and propagation for $k>\pi / d$. In contrast, if $\varepsilon<0$, propagation is allowed when $k<\pi / d$ (and forbidden for $k>\pi / d$ ). Within this passband a backward wave $(d q / d \omega<0)$ propagates, as one can see in Fig. 2 (thin solid lines).

This amazing behavior disappears if one fills the waveguide with the analyzed nonlocal wire medium. Using Eq. (7), we have in this case

$$
q_{x}^{2}+q_{z}^{2}=k^{2}-k_{0}^{2}, \quad q_{x}=\sqrt{k^{2}-k_{0}^{2}-\left(\frac{\pi}{d}\right)^{2}},
$$

and we obtain the usual frequency behavior: cutoff for $k$ $<\sqrt{(\pi / d)^{2}+k_{0}^{2}}$ and propagation for $k>\sqrt{(\pi / d)^{2}+k_{0}^{2}}$. An increase of the cutoff frequency is observed compared to the 
case when there is no filling medium, as one can see in Fig. 2 (thick solid line and dashed line).

Let us finally analyze the propagation of plane waves along the wire medium for the particular case of $q_{z}=k$. In this case, the nonlocal permittivity along the $z$ axis (6) becomes infinite. To avoid the singularity problem we use material equation of the form $\mathbf{E}=\overline{\bar{\varepsilon}}^{-1} \mathbf{D}$. In this case, the Maxwell equations have plane-wave solutions for all frequencies. For these waves the transverse wave vector $\mathbf{q}_{\perp}=\left(q_{x}, q_{y}\right)^{\mathrm{T}}$ is arbitrary. The waves are transverse with respect to the wire axis: $H_{z}=0$ and $E_{z}=0$. The electric field is parallel to the transverse wave vector, $\mathbf{q}_{\perp} \times \mathbf{E}=\mathbf{0}$.

Such waves can be interpreted as transmission-line modes propagating along the parallel wires. In fact, a set of $N$ infinite parallel wires can be viewed as a system of coupled transmission lines. This system can support $N$ degenerate transmission-line waves with $H_{z}=0, E_{z}=0$, and phase constant $q_{z}=k$. The electric field of these waves can be obtained from

$$
\mathbf{E}=-\left(\mathbf{u}_{\mathbf{x}} \partial_{x}+\mathbf{u}_{\mathbf{y}} \partial_{y}\right) \phi(x, y) \exp (-j k z),
$$

where $\phi(x, y)$ is a quasielectrostatic potential taking constant but arbitrary values $\phi_{n}(n=1,2, \ldots, N)$ at each wire. In fact, the plane wave with transverse wave number $\mathbf{q}_{\perp}$ and $q_{z}=k$ corresponds to the transmission-line wave with $\phi_{n}$ $=\phi_{0} \exp \left(-j \mathbf{q}_{\perp} \cdot \mathbf{r}_{n}\right)$, where $\mathbf{r}_{n}=\left(x_{n}, y_{n}\right)^{\mathrm{T}}$ is the location of the $n$th wire in the transverse $x-y$ plane.

In summary, it has been shown that parallel wire media possess very strong spatial dispersion effects at any frequencies, including the very large wavelength limit. An analytical model for the nonlocal permittivity dyadic of these media has been presented and discussed. Inconsistency of the local model for parallel wire media with nonvanishing wavevector component along the wires has been shown. Dramatic differences in the predicted behavior of that media, arising from the use of the conventional local and/or the nonlocal model for the permittivity are shown. Finally, the proposed nonlocal model for the permittivity has been found to be also suitable for the description of the transmission-line modes of the structure. We feel that the reported results open the question of the role of spatial dispersion in the adequate characterization of discrete metamaterials as effective media, at least if arbitrary directions of propagation and/or polarization of the electromagnetic field should be considered in the analysis. In addition, an example has been presented of an effective medium in which spatial dispersion is important at any frequency, in contrast with some commonly assumed ideas about the physical relevance of this effect.
${ }^{1}$ L. Landau, E. Lifschitz, and L. Pitaevski, Electrodynamics of Continuous Media (Pergamon Press, Oxford, 1984).

${ }^{2}$ J. Brown, Prog. Dielectr. 2, 195 (1960).

${ }^{3}$ W. Rotman, IRE Trans. Antennas Propag. 10, 82 (1962).

${ }^{4}$ R. King, D. Thiel, and K. Park, IEEE Trans. Antennas Propag. 31, 471 (1983).

${ }^{5}$ S. Maslovski, S. Tretyakov, and P. Belov, Microwave Opt. Technol. Lett. 35, 47 (2002).

${ }^{6}$ J. Pendry, A. Holden, W. Steward, and I. Youngs, Phys. Rev. Lett. 76, 4773 (1996).

${ }^{7}$ J. Pitarke, F. Garcia-Vidal, and J. Pendry, Phys. Rev. B 57, 15261 (1998).

${ }^{8}$ V. Veselago, Sov. Phys. Usp. 10, 509 (1968), [Usp. Fiz. Nauk 92, 517 (1967)].
${ }^{9}$ J. Pendry, Phys. Rev. Lett. 85, 3966 (2000).

${ }^{10}$ D. Smith, W. Padilla, D. Vier, S. Nemat-Nasser, and S. Schultz, Phys. Rev. Lett. 84, 4184 (2000).

${ }^{11}$ R. Shelby, D. Smith, and S. Schultz, Science 292, 77 (2001).

${ }^{12}$ D.R. Smith and N. Knoll, Phys. Rev. Lett. 85, 2933 (2000).

${ }^{13}$ A. Pokrovsky and A. Efros, Phys. Rev. Lett. 89, 093901 (2002).

${ }^{14}$ V. Ginzburg, The Propagation of Electromagnetic Waves in Plasmas (Pergamon, Oxford, 1964).

${ }^{15}$ I.V. Lindell, S. Tretyakov, K. Nikoskinen, and S. Ivonen, Microwave Opt. Technol. Lett. 31, 129 (2001).

${ }^{16}$ P. Belov, S. Tretyakov, and A. Viitanen, J. Electromagn. Waves Appl. 16, 1153 (2002). 